\title{
AUTOEFICÁCIA DO ALEITAMENTO MATERNO EM PUÉRPERAS DE UMA MATERNIDADE PÚBLICA DO NORDESTE BRASILEIRO
}

\section{SELF-EFFICACY OF BREASTFEEDING IN POSTPARTUM WOMEN ASSISTED IN A PUBLIC MATERNITY IN NORTHEASTERN BRAZIL}

\section{AUTOEFECTIVIDAD DE LA LACTANCIA EN MUJERES EMBARAZADAS DE UNA MATERNIDAD PÚBLICA EN EL NORESTE DE BRASIL}

Floriacy Stabnow Santos ${ }^{1}$, Rafael Cruz Souza², Paula Gabrielle Gomes Candido ${ }^{3}$, Leonardo Hunaldo dos Santos ${ }^{4}$, Livia Maia Pascoal5, Marcelino Santos Neto ${ }^{6}$

\section{RESUMO}

Objetivo: Verificar associação entre variáveis de contextos sociodemográfico e obstétrico com a autoeficácia em amamentar de puérperas residentes no Nordeste brasileiro. Método: Estudo transversal quantitativo realizado em maternidade pública de referência, com 160 mulheres que estavam no puerpério imediato até o 600 dia pós-parto. Dados coletados por meio de entrevistas individuais aplicando-se um formulário para obter informações acerca das características sociodemográficas e obstétricas, além da aplicação da escala de autoeficácia em aleitamento materno, na versão em português. Resultados: Mulheres com idade entre 26 e 35 anos tiveram quase 13 vezes mais chance de praticar alta autoeficácia em amamentar e mulheres com mais de 35 anos tiveram 21 vezes mais chance de ter maior alta autoeficácia. Mulheres que cursaram o ensino fundamental tiveram 39 vezes mais chance de ter maior alta autoeficácia e multíparas tiveram 4,44 mais chance de ter maior alta autoeficácia. Denotou-se que as puérperas tiveram alta autoeficácia para amamentar. Conclusões: A idade materna, a escolaridade, a situação obstétrica mostraram-se estatisticamente significativas para a alta autoeficácia do aleitamento materno, o que demonstra um panorama favorável ao aleitamento materno, sendo necessário acompanhamento do profissional enfermeiro para garantir assistência mais efetiva no apoio à amamentação.

Descritores: Aleitamento Materno; Autoeficácia; Nutrição Materna; Enfermagem.

\begin{abstract}
Objective: To verify the association between variables of sociodemographic and obstetric contexts with the self-efficacy in breastfeeding of postpartum women living in Northeast Brazil. Method: Quantitative cross-sectional study carried out in a public maternity of reference, with 160 women who were in the immediate puerperium until the $60^{\text {th }}$ postpartum day. Data collected through individual interviews using a form to obtain information about sociodemographic and obstetric characteristics, in addition to the application of the self-efficacy scale in breastfeeding, in the Portuguese version. Results: Women aged between 26 and 35 years were almost 13 times more likely to practice high self-efficacy in breastfeeding and women over 35 years were 21 times more likely to have greater high self-efficacy. Women who attended elementary school were 39 times more likely to have greater high self-efficacy and multiparous women were 4.44 more likely to have greater high self-efficacy. It was noted that mothers had high self-efficacy to breastfeed. Conclusions: Maternal age, education, obstetric situation were statistically significant for the high self-efficacy of breastfeeding, which demonstrates a favorable scenario for breastfeeding, requiring monitoring by the professional nurses to ensure more effective assistance support breastfeeding.
\end{abstract}

Descriptors: Breastfeeding; Self-efficacy; Maternal Nutrition; Nursing.

\section{RESUMEN}

Objetivo: Verificar la asociación entre variables de contextos sociodemográficos y obstétricos con la autoeficacia en la lactancia materna de mujeres puerperas que viven en el noreste de Brasil. Método: Estudio cuantitativo transversal realizado en un hospital público de maternidad de referencia, con 160 mujeres que estuvieron en el puerperio inmediato hasta el día 60 posparto . Datos recopilados a través de entrevistas individuales utilizando un formulario para obtener información sobre las características sociodemográficas y obstétricas, además de la aplicación de la escala de autoeficacia en la lactancia materna en la versión portuguesa. Resultados: Las mujeres de entre 26 y 35 años tenían casi 13 veces más probabilidades de practicar una alta autoeficacia en la lactancia materna y las mujeres mayores de 35 años tenían 21 veces más probabilidades de tener una mayor autoeficacia. Las mujeres que fueron a la escuela primaria tenían 39 veces más probabilidades de tener una mayor autoeficacia y las mujeres multíparas tenían 4,44 más probabilidades de tener una autoeficacia alta. Se observó que las madres tenían una alta autoeficacia para amamantar. Conclusiones: La edad materna, la educación, la situación obstétrica fueron estadísticamente significativas para la alta autoeficacia de la lactancia materna, lo que demuestra un escenario favorable para la lactancia materna, que requiere el seguimiento de la enfermera profesional para garantizar una asistencia más efectiva en el apoyo a la lactancia materna.

Descriptores: Lactancia Materna; Autoeficacia; Nutrición Materna; Enfermería.

${ }^{1}$ Enfermeira. Doutora em Ciências. Professora da Universidade Federal do Maranhão. ${ }^{2}$ Enfermeiro graduado pela Universidade Federal do Maranhão. ${ }^{3}$ Enfermeira. Discente da Pós-graduação em Saúde e Tecnologia da Universidade Federal do Maranhão. ${ }^{4}$ Biólogo. Doutor em Melhoramento Genético. Professor da Universidade Federal do Maranhão. ${ }^{5}$ Enfermeira. Doutora em Enfermagem. Professora da Universidade Federal do Maranhão. ${ }^{6}$ Farmacêutico bioquímico. Doutor em Ciências. Professor da Universidade Federal do Maranhão.

Como citar este artigo:

Santos FS, Souza RC, Candido PGG, et al. Autoeficácia do aleitamento materno em puérperas de uma maternidade pública do nordeste brasileiro. Revista de Enfermagem do Centro-Oeste Mineiro. 2020;10:e3910. [Access ]; Available in: DOI: 


\section{INTRODUÇÃO}

A amamentação é um processo dinâmico, que envolve um vínculo forte entre mãe e filho, indo além do aspecto da nutrição. Essa prática tem inúmeros benefícios para a saúde física e psíquica de mães e crianças $^{(1)}$. $O$ ato de amamentar estimula o desenvolvimento cognitivo e protege os bebês de infecções diarreicas e pneumonia e contribui para diminuir o risco de obesidade e doenças crônicas. Serve ainda como proteção contra o câncer de ovário e de mama, entre as nutrizes, e como anticoncepcional comportamental(2).

A Organização Mundial da Saúde (OMS) recomenda o aleitamento materno exclusivo (AME) até o sexto mês de vida da criança, isto é: sem água, chá, sucos, sopas ou papinhas. Depois disso, outros alimentos devem ser incluídos, mas a recomendação é que a amamentação seja mantida até os 2 anos de idade ou mais ${ }^{(1)}$.

Ainda, a amamentação resulta em menores custos com saúde. Estima-se que o aumento da amamentação poderia prevenir 823 mil mortes anuais em crianças menores de 5 anos, ou 13,8\% de todas as mortes de crianças menores de 24 meses e 20 mil mortes anuais por câncer de $\operatorname{mama}^{(3)}$.

As prevalências dos indicadores de aleitamento materno (AM) e AME no Brasil nas últimas três décadas apresentaram tendência ascendente, observada entre 1986 e 2006, sendo que teve uma relativa estabilização em 2013. Já o AM continuado até o segundo ano manteve-se estável entre 1986 e 2006, sendo o único indicador com aumento da prevalência entre 2006 e $2013^{(4)}$. A prevalência do AME em menores de 6 meses foi de $42,9 \%$ em São Luís, capital do Maranhão(5), em pesquisa realizada em 2015, e pesquisa anterior realizada em Imperatriz (MA) mostrara prevalência do AME em menores de 6 meses de $32 \%{ }^{(6)}$.

Apesar das investigações científicas desenvolvidas nas últimas décadas servirem para embasar a atual política de AM no Brasil e contribuírem para o desenvolvimento do conhecimento acerca dos benefícios dessa prática, além de sua importância para o contexto social e sua eficiência na diminuição da morbimortalidade infantil ${ }^{4}$, percebem-se ainda taxas reduzidas de amamentação.

Há eficácia e permanência da amamentação associada com maior autoconfiança da mulher nutriz em amamentar. Além disso, existem fatores que predispõem ao desmame precoce, como problemas com as mamas, ingurgitamento mamário, mastite, fissura ou ferida mamilar, bem como dor e formação de abscessos mamários, que podem ser identificados ainda na maternidade ${ }^{(7)}$.

Outro ponto refere-se ao nível de conhecimento sobre amamentação e fatores sociodemográficos influenciar no processo de amamentação. Quanto maior o nível de conhecimentos sobre AM, melhor a disposição e a intenção materna para amamentar após o parto ${ }^{(8)}$.

A teoria da aprendizagem social(9) destaca que a confiança ou a autoeficácia norteiam os comportamentos de saúde das pessoas, de forma que, quando elas aderem a comportamentos saudáveis, elas se esforçam e os alcançam. Assim, se a mulher acredita que ela é capaz de amamentar (autoeficácia), ela tem mais chance de amamentar com mais efetividade, e essa conscientização deve ocorrer antes que a amamentação seja empreendida ${ }^{(9)}$.

Como o comportamento da mulher diante do AM ainda não havia sido estudado dentro da perspectiva da autoeficácia, estudiosos do assunto $^{(10)}$ elaboraram uma escala para avaliar a confiança da mãe na amamentação. Essa escala de autoeficácia, denominada Breastfeeding SelfEfficacy Scale (BSES), já foi aplicada entre mulheres adolescentes no Canadá(11), mulheres africanas $^{(12)}$, mulheres espanholas ${ }^{(13)}$ e mulheres no sul do Brasil(14).

A BSES permite que o profissional de saúde conheça com antecedência a área em que a mulher tem menor autoeficácia e utilize tal informação como subsídio para o desenvolvimento de estratégias de apoio e promoção ao AM voltado para cada mulher, de forma individualizada, antes que ela decida não amamentar ou desmamar a criança precocemente. Em consequência, esperam-se a redução das taxas de desmame precoce e melhor saúde para a criança.

Nesse contexto, com este estudo, objetivou-se verificar a associação entre variáveis de contextos sociodemográfico e obstétrico com a autoeficácia em amamentar de puérperas.

\section{MÉTODOS}

Estudo transversal realizado no Hospital Regional Materno Infantil de Imperatriz (HRMI), instituição de referência para toda a região sudoeste do estado do Maranhão, que atende à clientela de municípios e estados circunvizinhos. A pesquisa foi realizada entre julho e dezembro de 2018. 
Mensalmente, são realizados, em média, 430 procedimentos entre partos normais e cirúrgicos. Considerando um intervalo com $90 \%$ de confiança, erro amostral de 5\%, a amostra compreendeu 160 puérperas, tendo sido adotado o critério da conveniência para a seleção das participantes. Foram incluídas mulheres que estavam no puerpério imediato até o 60 dia pósparto que tiveram seus filhos no HRMI, residentes em Imperatriz ou região e que estavam em AM. Foram excluídas puérperas, com problemas mentais ou cognitivos que impedissem a comunicação com os pesquisadores.

O convite para participar da pesquisa foi realizado no momento em que a mulher comparecia no ambulatório da instituição, para realizar a consulta de acompanhamento da criança. A coleta de dados ocorreu por meio de entrevistas individuais, aplicando-se um formulário para obter informações acerca das características sociodemográficas (idade, escolaridade, renda familiar e situação laboral) e obstétricas (local de pré-natal, número de consultas e abortos), das dificuldades enfrentadas, durante a amamentação e da condição de AM.

Aplicou-se ainda o instrumento BSES na versão em português, para classificar a autoeficácia em AM. Tal escala é do tipo Likert, contém 14 itens divididos em domínios técnico e pensamento intrapessoal, e cada questão apresenta cinco possibilidades de resposta, com escores variando de 1 a $5^{(15)}$. A cada item são atribuídas pontuações (1 equivale a discordo totalmente e 5 significa concordo totalmente), de modo que o escore total varia de 14 a 70 pontos, com a seguinte classificação: 14 a 32 pontos corresponde à baixa autoeficácia; 33 a 51 pontos à média autoeficácia e 52 a 70 pontos à alta

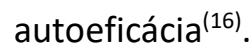

Os dados coletados foram armazenados em um banco de dados específico criado no programa Microsoft Excel versão 2016. Após a verificação de erros e inconsistências, foi realizada uma análise descritiva por meio de frequências relativas e absolutas de todas as características estudadas.

Para avaliar possíveis associações entre a autoeficácia em amamentar e variáveis sociodemográficas e obstétricas, foram utilizados o teste qui-quadrado com e sem correção de continuidade de Yates, o teste exato de Fisher ou o teste de Fisher-Freeman-Halton, dependendo do comportamento dos dados. Para associações 2x2 significativas, estimaram-se, ainda, razões de chance (RC), considerando intervalo de confiança de $95 \%$.

Todos os testes foram realizados no programa IBM Statistical Package for Social Science (SPSS), versão 24, a 5\% de significância.

A pesquisa atendeu aos preceitos éticos e foi aprovada pelo Comitê de Ética em pesquisa da Universidade Federal do Maranhão, sob o parecer 935.114.

\section{RESULTADOS E DISCUSSÃO}

A maioria das mulheres tinha idade entre 26 e 35 anos (75,63\%), mas não foram registradas mulheres com idades entre 20 e 25 anos. Elas cursaram o Ensino Médio (60,62\%), possuíam renda mensal entre um e dois salários mínimos $(70,63 \%)$ e eram donas de casa $(73,75 \%)$. Os antecedentes obstétricos mostraram que eram primíparas $(58,13 \%)$, realizaram o pré-natal na Unidade Básica de Saúde (UBS) (63,75\%), passaram por seis ou mais consultas $(76,88 \%)$ e não tiveram histórico de abortos (88,13\%). Ainda, a maioria das puérperas não apresentou dificuldades para amamentar $(63,75 \%)$ e praticava AME $(77,50 \%)$. Leite em pó foi citado como alimento complementar mais utilizado pela maior parte das entrevistadas. Com a aplicação da escala, a maioria absoluta das puérperas apresentou alta autoeficácia em amamentar $(86,25 \%)$, seguida da média autoeficácia $(13,75 \%)$ não havendo registros de baixa autoeficácia (Tabela 1).

Tabela 1 - Perfil sociodemográfico e obstétrico de puérperas, dificuldades para amamentar e alimentos e líquidos oferecidos, Imperatriz (MA), Brasil, 2018.

\begin{tabular}{lc}
\hline \hline Variáveis & $\mathbf{n}(\%)$ \\
\hline Idade da mãe, anos & \\
$<19$ & $21(13,12)$ \\
$26-35$ & $121(75,63)$ \\
$>35$ & $18(11,25)$ \\
\hline
\end{tabular}




\begin{tabular}{|c|c|}
\hline Variáveis & $\mathrm{n}(\%)$ \\
\hline \multicolumn{2}{|l|}{ Escolaridade } \\
\hline Sem instrução & $2(1,25)$ \\
\hline Ensino Fundamental & $54(33,75)$ \\
\hline Ensino Médio & $97(60,62)$ \\
\hline Ensino Superior & $7(4,38)$ \\
\hline \multicolumn{2}{|c|}{ Renda familiar, salário mínimo* } \\
\hline$<1$ & $29(18,13)$ \\
\hline $1-2$ & $113(70,63)$ \\
\hline 3 ou mais & $18(11,25)$ \\
\hline \multicolumn{2}{|l|}{ Situação laboral } \\
\hline Dona de casa & $118(73,75)$ \\
\hline Trabalha fora & $42(26,25)$ \\
\hline \multicolumn{2}{|l|}{ Situação obstétrica } \\
\hline Primípara & $93(58,13)$ \\
\hline Multípara & $67(41,88)$ \\
\hline \multicolumn{2}{|l|}{ Pré-natal } \\
\hline UBS & $102(63,75)$ \\
\hline HRMI & $46(28,75)$ \\
\hline Outro local & $11(6,88)$ \\
\hline Não fez pré-natal & $1(0,63)$ \\
\hline \multicolumn{2}{|l|}{ Número de consultas } \\
\hline$<6$ & $37(23,13)$ \\
\hline 6 ou mais & $123(76,88)$ \\
\hline \multicolumn{2}{|l|}{ Aborto } \\
\hline Não teve aborto & $141(88,13)$ \\
\hline 1 aborto & $13(8,13)$ \\
\hline 2 abortos & $6(3,75)$ \\
\hline \multicolumn{2}{|l|}{ Dificuldades para amamentar } \\
\hline Sem dificuldades & $102(63,75)$ \\
\hline Problemas no mamilo & $23(14,36)$ \\
\hline Pouco leite & $22(13,75)$ \\
\hline Pega incorreta & $10(6,25)$ \\
\hline Problema bebê & $1(0,63)$ \\
\hline Prematuridade & $1(0,63)$ \\
\hline Leite fraco & $1(0,63)$ \\
\hline \multicolumn{2}{|l|}{ Aleitamento complementar } \\
\hline Leite em pó & $22(13,75)$ \\
\hline Água & $4(2,50)$ \\
\hline Chá & $4(2,50)$ \\
\hline Leite em pó + água & $2(1,25)$ \\
\hline Papinha industrializada & $1(0,63)$ \\
\hline Amido de milho & $1(0,63)$ \\
\hline Leite soja & $1(0,63)$ \\
\hline Massa de macaxeira & $1(0,63)$ \\
\hline \multicolumn{2}{|l|}{ AME } \\
\hline Sim & $124(77,50)$ \\
\hline Não & $36(22,50)$ \\
\hline \multicolumn{2}{|l|}{ Autoeficácia } \\
\hline Média & $22(13,75)$ \\
\hline Alta & $138(86,25)$ \\
\hline
\end{tabular}

Fonte: Elaborada pelos próprios autores

UBS: Unidade Básica de Saúde; HRMI: Hospital Regional Materno Infantil de Imperatriz; AME: aleitamento materno exclusivo. * Valor do salário mínimo $\mathrm{R} \$ 937,00$.

O perfil sociodemográfico e econômico da mãe pode representar influência na amamentação da criança. Estudo sobre amamentação realizado em Belo Horizonte (MG) ${ }^{17}$ também constatou que a maior concentração de mulheres estava em faixa etária semelhante e tinham pouca escolaridade. A idade materna e a escolaridade podem influenciar na decisão da mãe amamentar ou não. Quanto menor for a idade materna e a escolaridade, mais dificuldades e inexperiência esta deve ter no processo de cuidado e no aleitamento de seu filho ${ }^{(18)}$.

Sobre a renda familiar, houve predomínio de mulheres vivendo com renda entre um e dois salários mínimos. Estudo realizado em Pernambuco também demonstrou que a maioria das puérperas tinha renda de um salário mínimo. o nível socioeconômico está diretamente 
associado com a amamentação, visto que as mulheres que apresentam melhor situação econômica inclinam-se a amamentar por períodos mais amplos ${ }^{(18)}$.

Na presente casuística, 118 mulheres eram donas de casa, situação considerada positiva para a prática da amamentação, já que mães que trabalham fora de casa apresentam mais chances de desmamar precocemente seus filhos. 0 trabalho da mulher fora de casa pode dificultar muito a rotina de amamentação(15). A ocupação materna pode estar associada ao desmame precoce, uma vez que ter uma atividade remunerada e sair para trabalhar pode dificultar a amamentação ${ }^{(19)}$. As participantes deste estudo demonstraram situação favorável à prática da amamentação.

O pré-natal representa um impacto sobre a assistência à saúde de mulheres gestantes. No presente estudo, $63,7 \%$ das mulheres pesquisadas realizaram o pré-natal na UBS. Essas unidades devem funcionar como porta de entrada para o sistema de saúde, garantindo resolutividade e atendimento integral ${ }^{(20)}$, com foco na promoção do AM e na prevenção de agravos que podem ser evitados com a prática do AME.

Um dado relevante para o sucesso do prénatal é o número de consultas. Observou-se que $76,9 \%$ realizaram seis ou mais consultas no prénatal. Esse é o momento mais oportuno para promover a cultura do AM com 0 desenvolvimento de ações educativas direcionadas para a mulher, visando ao sucesso dessa prática. Geralmente, é durante a gestação que a mulher decide se vai ou não amamentar. Assim, as consultas de pré-natal devem ser permeadas de orientações sobre a importância do $\mathrm{AM}^{(20)}$.

Sobre as dificuldades encontradas pelas mães para amamentar, $63,8 \%$ não referiram dificuldades, mas as demais alegaram algum tipo de dificuldade, como problemas nos mamilos, pouco leite, pega incorreta, problemas com o bebê e leite fraco. A pega incorreta pode levar o bebê a rejeitar o peito da mãe, mas isso pode estar relacionado a fatores como bebê mal posicionado, com pescoço e/ou cabeça girada, desalinhado com o corpo, não apoiado, com o queixo longe da mama, mais aréola vista abaixo do lábio do bebê, lábios invertidos, boca não completamente aberta, sucções rápidas e superficiais, mãe interrompe a mamada, ou ainda mamas duras e brilhantes após a mamada e sem sinal do reflexo da ocitocina, os quais apontam erro de técnica, trazendo agravos à boa qualidade do $\mathrm{AM}^{(20,21)}$.

Além do leite materno, as crianças do presente estudo receberam outros complementos, como chá $(2,5 \%)$, água $(2,5 \%)$, leite em pó $(13,75 \%)$, leite de soja $(0,63 \%)$ e papinha industrializada $(0,63 \%)$. O AME é fator protetor contra doenças infecciosas. Estudo realizado no interior do Maranhão mostrou que crianças que usaram água, chá, leites artificiais e mingaus foram mais afetadas por diarreias do que as que estavam em $\mathrm{AME}^{(5)}$.

Evidenciou-se, neste estudo, que a maioria das puérperas apresentou alta autoeficácia em amamentar (86,3\%). Estudo realizado em Recife (PE) com 132 gestantes mostrou que a autoeficácia materna para amamentar pode ser modificada ou consolidada quando se usam intervenções educativas que visam a melhorar $\mathrm{e}$ superar as dificuldades iniciais da mulher no processo da lactação ${ }^{(22)}$.

$\mathrm{Na}$ análise bruta, as variáveis idade entre 15 e 19 anos e mais que 35, escolaridade Ensino Fundamental, situação obstétrica multípara, estar em AME e aleitamento complementar apresentaram associação estatisticamente significante $(p<0,20)($ Tabela 2$)$.

Tabela 2 - Análise de regressão logística univariada dos casos de alta autoeficácia em puérperas, Imperatriz (MA), Brasil, 2018.

\begin{tabular}{lccc}
\hline \hline Variáveis & $\begin{array}{c}\text { Autoeficácia alta } \\
\mathbf{n}(\%)\end{array}$ & Razão de chance (IC95\%) & Valor de $\mathbf{p} *$ \\
\hline Idade mãe, anos & $13(61,9)$ & 1,00 & 0,002 \\
$\quad<19$ & $108(89,3)$ & $5,11(1,78-14,64)$ & 0,01 \\
$26-35$ & $17(94,4)$ & $10,46(1,16-94,48)$ & \\
$\quad>35$ & $1(50,0)$ & 1,00 & 0,13 \\
Escolaridade & $49(90,7)$ & $9,80(0,53-181,80)$ &
\end{tabular}




\begin{tabular}{|c|c|c|c|}
\hline Variáveis & $\begin{array}{c}\text { Autoeficácia alta } \\
\text { n (\%) }\end{array}$ & Razão de chance (IC95\%) & Valor de $\mathrm{p}^{*}$ \\
\hline Ensino Médio & $81(83,5)$ & $5,06(0,30-85,21)$ & 0,26 \\
\hline Ensino Superior & $7(100,0)$ & + & - \\
\hline \multicolumn{4}{|c|}{ Renda familiar, salário mínimo } \\
\hline$<1$ & $23(79,3)$ & 1,00 & \\
\hline $1-2$ & $99(87,6)$ & $1,85(0,64-5,32)$ & 0,26 \\
\hline 3 ou mais & $16(88,9)$ & $2,09(0,37-11,69)$ & 0,40 \\
\hline \multicolumn{4}{|l|}{ Situação laboral } \\
\hline Dona de casa & $101(85,6)$ & 1,00 & \\
\hline Trabalha fora & $37(88,1)$ & $1,25(0,43-3,62)$ & 0,69 \\
\hline \multicolumn{4}{|l|}{ Situação obstétrica } \\
\hline Primípara & $74(79,6)$ & 1,00 & \\
\hline Multípara & $64(95,5)$ & $5,48(1,55-19,36)$ & 0,008 \\
\hline \multicolumn{4}{|l|}{ Pré-natal } \\
\hline Não fez pré-natal & $1(100,0)$ & + & \\
\hline HRMI & $40(87,0)$ & $0,67(0,07-6,20)$ & 0,72 \\
\hline UBS & $87(85,3)$ & $0,58(0,07-4,87)$ & 0,62 \\
\hline Outro local & $10(90,9)$ & 1,00 & \\
\hline \multicolumn{4}{|l|}{ Número de consultas } \\
\hline$<6$ & $31(83,8)$ & 1,00 & \\
\hline 6 ou mais & $107(87,0)$ & $1,29(0,47-3,59)$ & 0,62 \\
\hline \multicolumn{4}{|l|}{ Abortos } \\
\hline Não teve aborto & $121(85,8)$ & $1,21(0,13-0,90)$ & 0,87 \\
\hline 1 aborto & $12(92,3)$ & $2,40(0,12-46,39)$ & 0,56 \\
\hline 2 abortos & $5(83,3)$ & 1,00 & \\
\hline \multicolumn{4}{|l|}{ Dificuldades do aleitamento } \\
\hline Sim & $90(88,2)$ & $1,56(0,63-3,88)$ & 0,34 \\
\hline Não & $48(82,8)$ & 1,00 & \\
\hline \multicolumn{4}{|l|}{ Dificuldades } \\
\hline Problemas no mamilo & $13(81,3)$ & 1,00 & \\
\hline Pouco leite & $18(81,8)$ & $1,04(0,20-5,45)$ & 0,96 \\
\hline Leite fraco & $1(100,0)$ & + & - \\
\hline Sem dificuldades & $90(88,2)$ & $1,73(0,43-6,97)$ & 0,44 \\
\hline Pega incorreta & $8(80,0)$ & $0,92(0,13-6,78)$ & 0,94 \\
\hline Problemas no mamilo & $6(85,7)$ & $1,39(0,12-16,23)$ & 0,80 \\
\hline Prematuridade & $1(100,0)$ & $t$ & - \\
\hline Problema bebê & $1(100,0)$ & + & - \\
\hline \multicolumn{4}{|l|}{ Alimento complementar } \\
\hline Leite em pó & $14(63,6)$ & 1,00 & \\
\hline Leite em pó + água & $1(50,0)$ & $0,57(0,03-10,44)$ & 0,71 \\
\hline Chá & $2(50,0)$ & $0,57(0,07-4,88)$ & 0,61 \\
\hline Água & $3(75,0)$ & $1,71(0,15-19,36)$ & 0,66 \\
\hline Massa de macaxeira & $1(100,0)$ & + & - \\
\hline Leite soja & $0(0,0)$ & $\dagger$ & - \\
\hline Amido de milho & $1(100,0)$ & $\dagger$ & - \\
\hline Papinha industrializada & $1(100,0)$ & + & - \\
\hline \multicolumn{4}{|l|}{ AME } \\
\hline Sim & $115(92,7)$ & $7,22(2,76-18,87)$ & 0,001 \\
\hline Não & $23(63,9)$ & 1,00 & \\
\hline
\end{tabular}

Fonte: Elaborada pelos próprios autores

*Qui-quadrado de Wald; †presença de valores zerados que impossibilitam o cálculo da razão de chance.

IC95\%: intervalo de 95\% de confiança; UBS: Unidade Básica de Saúde; HRMI: Hospital Regional Materno Infantil de Imperatriz; AME: aleitamento materno exclusivo.

No modelo final de regressão (análise ajustada), permaneceram associadas à alta autoeficácia em amamentar $(p<0,05)$ as variáveis idade entre 15 e 19 anos e maior que 35, escolaridade Ensino Fundamental, situação obstétrica multípara, estar em AME e aleitamento complementar (Tabela 3). 
Tabela 3 - Análise multivariada (ajustada) dos casos alta autoeficácia em puérperas, Imperatriz (MA), Brasil, 2018.

\begin{tabular}{|c|c|c|c|}
\hline Variáveis & Razão de chance & IC95\% & Valor de $\mathrm{p}^{*}$ \\
\hline \multicolumn{4}{|l|}{ Idade mãe, anos } \\
\hline$<19$ & 1,00 & & \\
\hline $26-35$ & 12,59 & $3,01-52,74$ & 0,001 \\
\hline$>35$ & 21,18 & $1,95-230,21$ & 0,01 \\
\hline \multicolumn{4}{|l|}{ Escolaridade } \\
\hline Sem instrução & 1,00 & & \\
\hline Ensino Fundamental & 39,13 & $1,65-927,38$ & 0,02 \\
\hline Ensino Médio & 7,18 & $0,40-128,44$ & 0,18 \\
\hline Ensino Superior & + & - & - \\
\hline \multicolumn{4}{|l|}{ Situação obstétrica } \\
\hline Primípara & 1,00 & & \\
\hline Multípara & 4,44 & $1,17-16,89$ & 0,03 \\
\hline \multicolumn{4}{|l|}{ Aleitamento complementar } \\
\hline Leite em pó & 1,00 & & \\
\hline Leite em pó + água & 0,90 & $0,05-17,04$ & 0,95 \\
\hline Chá & 0,90 & $0,10-8,05$ & 0,93 \\
\hline Água & 2,07 & $0,17-25,17$ & 0,57 \\
\hline Massa de macaxeira & + & + & - \\
\hline Leite soja & + & + & - \\
\hline Amido de milho & $\dagger$ & $\dagger$ & - \\
\hline Papinha industrializada & + & + & - \\
\hline \multicolumn{4}{|l|}{ AME } \\
\hline Sim & 6,33 & $2,36-16,99$ & 0,001 \\
\hline Não & 1,00 & & \\
\hline
\end{tabular}

Fonte: Elaborada pelos próprios autores

*Qui-quadrado de Wald; † presença de valores zerados que impossibilitam o cálculo da razão de chance.

IC95\%: intervalo de $95 \%$ de confiança; AME: aleitamento materno exclusivo.

A idade materna foi um fator que contribuiu para a alta autoeficácia do AM entre as puérperas estudadas. A análise multivariada mostrou que mulheres com idade entre 26 e 35 anos tiveram 12,59 mais chances de ter mais alta autoeficácia $(p=0,001)$, e mulheres com mais de 35 anos tiveram 21,18 mais chances de ter mais alta autoeficácia $(p=0,01)$.

A escolaridade também se demonstrou, estatisticamente, significativa. Mulheres que cursaram o Ensino Fundamental (até 8 anos de estudo) tiveram 39,13 mais chance de ter mais alta autoeficácia $(p=0,001)$, sendo a amamentação considerada um fator protetor.

Estudo realizado em Banco de Leite Humano, na cidade de São Paulo, com dados coletados de 1.673 prontuários de mulheres atendidas $^{(23)}$ e estudo realizado em Fortaleza (CE) com 132 puérperas ${ }^{(16)}$ corroboraram os resultados encontrados na presente pesquisa, onde mulheres que tinham até 8 anos de estudo tiveram maior autoeficácia em amamentar. Entretanto, há estudos ${ }^{(8,17)}$ que demonstram que mulheres que tiveram acima de 8 anos de estudo tiveram uma prática de amamentação mais duradoura, isto é, mulheres que tiveram mais acesso a informação praticaram o aleitamento materno exclusivo por mais tempo.

A situação obstétrica apontou associação entre a autoeficácia em amamentar. Mulheres multíparas tiveram 4,44 mais chances de ter maior alta autoeficácia $(p=0,03)$. As mães primíparas, na gravidez, no parto ou no puerpério, podem manifestar comportamentos e sentimentos que culminam no aparecimento de crises na vida pessoal e familiar, o que pode interferir na prática do $\mathrm{AM}^{(24)}$. Dessa forma, o enfermeiro deve apoiálas, agindo como facilitador atento aos indícios de suas necessidades de orientações e cuidados. 0 enfermeiro é o profissional que pode aconselhar e influenciar no comportamento da mãe, apoiandoa, desde o pré-natal e reforçando sua intenção de amamentar ${ }^{(15)}$.

A confiança no AM revela-se na crença ou expectativa da mulher de que ela possui conhecimentos e habilidades suficientes para amamentar o seu bebê com êxito(24). A alta autoeficácia da amamentação foi um fator que contribuiu para a prática do AME. Os resultados mostraram que mulheres que apresentaram alta autoeficácia em amamentar tiveram 6,33 mais chances $(p=0,001)$ de estar em AME. 
Assim, o apoio da família e da equipe de saúde é essencial para o sucesso do AM. Não só no pré-natal, mas também no puerpério, a equipe de saúde e, em especial, o enfermeiro pode usar tecnologias educativas visando à promoção e ao apoio ao $\mathrm{AM}$, possibilitando às mães que dúvidas sejam esclarecidas e que a prática do AM seja efetivada ${ }^{(17)}$.

Portanto, no puerpério imediato, a mulher deve ser acompanhada no alojamento conjunto e apoiada para a prática da lactação, para que tenha suporte em suas dificuldades, sendo direcionada para consultas de puericultura, visando a manter sua confiança e autoeficácia em amamentar. Assim, os profissionais da enfermagem precisam entender e apoiar a amamentação, contribuindo para o êxito dessa prática.

\section{CONCLUSÃO}

A maioria das mulheres do presente estudo estavam em idade fértil, cursaram o Ensino Médio, tinham renda familiar entre um e dois salários mínimos, fizeram o pré-natal e eram donas de casa e primíparas.

Idade materna, escolaridade e situação obstétrica mostraram-se, estatisticamente, significativas para a alta autoeficácia do aleitamento materno, e esta se mostrou estatisticamente significativa para a prática do aleitamento materno exclusivo.

A partir da aplicação da Breastfeeding SelfEfficacy Scale, as mulheres do estudo apresentaram alta autoeficácia em amamentar, o que demonstra panorama favorável ao aleitamento materno, sendo necessário acompanhamento da equipe multiprofissional de saúde, para garantir assistência mais efetiva no apoio à amamentação. Entretanto, o fato de a avaliação de autoeficácia ter sido feita em apenas um momento pode consistir numa limitação, o que sugere a realização de pesquisas longitudinais que procurem a comparação de variáveis em períodos distintos no pós-parto.

Os achados contribuem para a compreensão do contexto sociodemográfico e obstétrico das puérperas na autoeficácia em amamentar, de forma que a mulher deve ser orientada e direcionada para consultas de puericultura, desde a alta hospitalar, evitando o desmame precoce.

\section{REFERÊNCIAS}

1 - Brasil. Ministério da Saúde. Saúde da criança: Aleitamento materno e alimentação complementar. Brasília: Ministério da Saúde; 2015.

2 - World Health Organization (WHO). United Nations Children's Fund (Unicef). Global breastfeeding scorecard; 2019. Increasing commitment to breastfeeding through funding and improved policies and programmes. Geneve: WHO/Unicef; 2019 [acesso em 28 jul 2020]. Disponível

em: https://apps.who.int/iris/bitstream/handle/1066 5/326049/WHO-NMH-NHD-19.22-eng.pdf?ua=1

3 - Victora CG, Bahl R, Barros AJ, França GV, Horton $S$, Krasevec J, et al. Breastfeeding in the 21st century: Epidemiology, mechanisms and lifelong effect. Lancet 2016;387(10017):475-90. DOI: 10.1016/S0140-6736(15)01024-7

4 - Boccolini CS, Boccolini PMM, Monteiro FR, Venâncio SI, Giugliani ERJ. Tendência de indicadores do aleitamento materno no Brasil em três décadas. Rev Saude Publica 2017;51:108. DOI: 10.11606/S1518-8787.2017051000029

5 - Rieth NFA, Coimbra LC. Caracterização do aleitamento materno em São Luís, Maranhão. Rev Pesq Saúde 2016 [acesso em 28 jul 2020]; 17(1):7$12 . \quad$ Disponível em: https://pdfs.semanticscholar.org/cf05/444d44d8 362c20bb5cea3c10a681b8ec86ca.pdf

6 -Santos FS, Santos LH, Saldan PC, Santos FC, Leite AM, Mello DF. Breastfeeding and acute diarrhea among children enrolled in the family health strategy. Texto Contexto-Enferm. 2016;25(1):1-8. DOI: $10.1590 / 0104-070720160000220015$

7 - Barbosa GE, Pereira JM, Soares MS, Pereira LB, Pinhon L, Caldeira AP. Initial difficulties with breastfeeding technique and the impact on duration of exclusive breastfeeding. Rev Bras Saúde Mater Infant. 2018;18(3):527-37. DOI: 10.1590/1806-93042018000300005

8 - Suárez-Cotelo MD, Movilla-Fernández MJ, PitaGarcía P, Fernández Arias B, Novío S. Breastfeeding knowledge and relation to prevalence. Rev Esc Enferm USP. 2019;53:e03433. DOI: $10.1590 / \mathrm{s} 1980-220 \times 2018004503433$

9 - Bandura A. Self-efficacy: toward a unifying theory of behavioral change. Psychol Rev. 1977; 84(2):191-215. DOI: 10.1016/01466402(78)90002-4

10 - Dennis CL, Faux S. Development and psychometric testing of the Breastfeeding SelfEfficacy Scale. Res Nurs Health. 1999; 22(5):399$409 . \quad$ DOI: $\quad 10.1002 /($ sici) $1098-$ 240x(199910)22:5<399::aid-nur6>3.0.co;2-4 11 - Dennis CL, Heaman MR, Mossman MR. Psychometric Testing of the Breastfeeding Self- 
Efficacy Scale-Short Form Among Adolescents. Journal of Adolescent Health. 2011;49:265-71. DOI: 10.1016/i.jadohealth.2010.12.015

12 - MCCarter-Spaulding D, Gore R. Breastfeeding self-efficacy in women of African descent. JOGNN. 2009;38(2):230-43. DOI: 10.1111/i.15526909.2009.01011.x

13 - Oliver-Roiga A, D’Anglade-González ML, Garcia-Garcia B, Silva-Tubio JR, Richart-Martinez $M$, Dennis $C L$. The Spanish version of the Breastfeeding Self-Efficacy Scale-Short Form: Reliability and validity assessment. Int J Nurs Stud. 2012;49:169-73.

DOI: 10.1016/i.ijnurstu.2011.08.005

14 - Zubaran C, Foresti K, Schumacher M, Thorell $M R$, Amoretti A, Muller $L$, et al. The Portuguese Version of the Breastfeeding Self-Efficacy ScaleShort Form. J Hum Lact. 2010;26(3):297-303. DOI: 10.1177/0890334409359916.

15 - Oriá MO, Ximenes LB. Tradução e adaptação cultural da Breastfeeding Self-Efficacy Scale para o português. Acta Paul Enferm. 2010;23(2):230-8. DOI: $10.1590 /$ S0103-21002010000200013

16 - Lopes BB, Lopes AF, Soares DG, Dodou HD, Castro RC, Oriá MO. Assessment of maternal selfefficacy in breastfeeding in the immediate puerperium. Rev Rene. 2017;18(6):818-24. DOI: 10.15253/2175-6783.2017000600016

17 - Souza SF, Alvarenga DB, Santos BN, Pinheiro IF, Salles PV. Perfil demográfico e levantamento dos conhecimentos sobre aleitamento materno de puérperas atendidas em uma maternidade pública da região metropolitana de Belo Horizonte: resultado de um projeto de extensão. Revista Interdisciplinar de Extensão 2017; 1(1)123-4. [acesso em 29 abr 2020]. Disponível em:http://periodicos.pucminas.br/index.php/con ecte-se/article/view/16195

18 - Cavalcanti SH, Caminha MF, Figueiroa JN, Serva VM, Cruz RS, Lira PI, et al. Fatores associados à prática do aleitamento materno exclusivo por pelo menos seis meses no estado de Pernambuco. Rev Bras Epidemiol. 2015;18(1):208-19. DOI: 10.1590/1980-5497201500010016

19 - Brecailo MK, Tamanini M. Amamentar, cuidar, maternar: regulações, necessidades e subjetividades. Demetra. 2016;11(3):825-46. DOI: 10.12957/demetra.2016.22507

20 - Silva DD, Schmitt IM, Costa R, Zampieri MF, Bohn IE, Lima MM. Promotion of breastfeeding in prenatal care: the discourse of pregnant women and Health professional. Rev Min Enferm. 2018; 22:e-1103. DOI: $10.5935 / 1415-2762.20180031$
21 - Souza SA, Araújo RT, Teixeira JR, Mota TN. Breastfeeding: factors affecting the early weaning between adolescent mothers. Journal of Nursing UFPE On Line. 2016; 10(10):3806-13. [acesso em 29 abr 2020]. Disponível em: https://periodicos.ufpe.br/revistas/revistaenferm agem/article/view/11446

22 - Javorski M, Rodrigues AJ, Dodt RC, Almeida PC, Leal LP, Ximenes LB. Effects of an educational technology on self-efficacy for breastfeeding and practice of exclusive breastfeeding. Rev Esc Enferm USP. 2018;52:e03329. DOI: 10.1590/S1980-220X2017031803329

23 - Carreiro JA, Amorim FA, Abrão AC, Marcacine KO, Abuchaim ES, Coca KP. Dificuldades relacionadas ao aleitamento materno: análise de um serviço especializado em amamentação. Acta Paul Enferm. 2018;31(4):430-8. DOI: 10.1590/1982-0194201800060

24 - Alves ID, Fretas AP, Abreu AC, Coelho MB, Peres TC. Abortamento espontâneo: vivência e significado em psicologia hospitalar. Revista Científica Semana Acadêmica. MMXVII;2017. acesso em 26 nov 2018]. Disponível em: https://semanaacademica.org.br/artigo/abortam ento-espontaneo-vivencia-e-significado-empsicologia-hospitalar

Nota: Este estudo é um recorte do Trabalho de Conclusão de Curso intitulado "Aplicação da versão brasileira da escala de autoeficácia do aleitamento materno entre mulheres no interior do Maranhão" para obtenção do título de Bacharel em Enfermagem do Curso de Enfermagem da Universidade Federal do Maranhão. "O presente trabalho foi realizado com apoio da Coordenação de Aperfeiçoamento de Pessoal de Nível Superior - Brasil (CAPES) - Código de Financiamento 001"

Recebido em: 08/08/2020

Aprovado em: 23/11/2020

Endereço de correspondência:

Floriacy Stabnow Santos

Universidade Federal do Maranhão - Campus Avançado Bom Jesus

Av. da Universidade, S/N, Bairro Dom Afonso Felipe Gregory Imperatriz-MA CEP: 65.915-240

E-mail: floriacys@gmail.com 